PROCEEDINGS OF THE

AMERICAN MATHEMATICAL SOCIETY

Volume 138, Number 6, June 2010, Pages 2025-2036

S 0002-9939(10)10232-9

Article electronically published on January 27, 2010

\title{
ON REGULARITY CRITERIA FOR WEAK SOLUTIONS TO THE MICROPOLAR FLUID EQUATIONS IN LORENTZ SPACE
}

\author{
BAOQUAN YUAN
}

(Communicated by Matthew J. Gursky)

\begin{abstract}
In this paper the regularity of weak solutions and blow-up criteria for smooth solutions to the micropolar fluid equations in three dimensional space are studied in the Lorentz space $L^{p, \infty}\left(\mathbb{R}^{3}\right)$. We obtain that if $u \in L^{q}\left(0, T ; L^{p, \infty}\left(\mathbb{R}^{3}\right)\right)$ for $\frac{2}{q}+\frac{3}{p} \leq 1$ with $3<p \leq \infty$ or if $\nabla u \in$ $L^{q}\left(0, T ; L^{p, \infty}\left(\mathbb{R}^{3}\right)\right)$ for $\frac{2}{q}+\frac{3}{p} \leq 2$ with $\frac{3}{2}<p \leq \infty$ or if the pressure $P \in L^{q}\left(0, T ; L^{p, \infty}\left(\mathbb{R}^{3}\right)\right)$ for $\frac{2}{q}+\frac{3}{p} \leq 2$ with $\frac{3}{2}<p \leq \infty$ or if $\nabla P \in$ $L^{q}\left(0, T ; L^{p, \infty}\left(\mathbb{R}^{3}\right)\right)$ for $\frac{2}{q}+\frac{3}{p} \leq 3$ with $1<p \leq \infty$, then the weak solution $(u, \omega)$ satisfying the energy inequality is a smooth solution on $[0, T)$.
\end{abstract}

\section{INTRODUCTION}

This paper concerns the regularity of weak solutions and blow-up criteria for smooth solutions to the micropolar fluid equations in three dimensions:

$$
\left\{\begin{array}{l}
\frac{\partial u}{\partial t}-(\mu+\chi) \Delta u+u \cdot \nabla u+\nabla P-\chi \nabla \times \omega=0, \\
\frac{\partial \omega}{\partial t}-\gamma \Delta \omega-\kappa \nabla \operatorname{div} \omega+2 \chi \omega+u \cdot \nabla \omega-\chi \nabla \times u=0, \\
\operatorname{div} u=0, \\
u(x, 0)=u_{0}(x), \omega(x, 0)=\omega_{0}(x),
\end{array}\right.
$$

where $u=\left(u_{1}(t, x), u_{2}(t, x), u_{3}(t, x)\right)$ denotes the velocity of the fluid at a point $x \in \mathbb{R}^{3}$ and time $t \in[0, T)$, and $\omega=\left(\omega_{1}(t, x), \omega_{2}(t, x), \omega_{3}(t, x)\right)$ and $P=P(t, x)$ denote, respectively, the micro-rotational velocity and the hydrostatic pressure. $u_{0}$ and $\omega_{0}$ are the prescribed initial data for the velocity and angular velocity, with $\operatorname{div} u_{0}=0 . \mu$ is the kinematic viscosity, $\chi$ is the vortex viscosity, and $\kappa$ and $\gamma$ are spin viscosities. A theory of micropolar fluids was first proposed by Eringen [8] in 1966, enabling us to consider some physical phenomena that cannot be treated by the classical Navier-Stokes equations for viscous incompressible fluids, for example, the motion of animal blood, liquid crystals and dilute aqueous polymer solutions,

Received by the editors August 20, 2009.

2010 Mathematics Subject Classification. Primary 35Q35, 76D03.

Key words and phrases. Micropolar fluid equations, regularity of weak solutions, Lorentz spaces.

The author was partially supported by the National Natural Science Foundation of China (grant No. 10771052), the Program for Science \& Technology Innovation Talents in Universities of Henan Province (grant No. 2009HASTIT007), the Doctor Fund of Henan Polytechnic University (grant No. B2008-62), and the Innovation Scientists and Technicians Troop Construction Projects of Henan Province. 
etc. The problems of existence of weak and strong solutions were treated by Galdi and Rionero [10] and Yamaguchi 30], respectively. If, further, the vortex viscosity $\chi=0$, then the velocity $u$ does not depend on the micro-rotation field $\omega$, and the first equation reduces to the classic Navier-Stokes equation, which has been intensively analyzed; see, for example, the classic books by Ladyzhenskaya [16, Lions [19] or Lemarié-Rieusset [18.

There is a vast literature on the mathematical theory of micropolar fluid equations (see, for example, [17, 30, 10, 9, 17, 29, 4, 5]). The existence and uniqueness of global solutions were extensively studied by Lange [17, Galdi and Rionero [10], and Yamaguchi [30]. Recently, Ferreira and Villamizar-Roa 9] considered the existence and stability of solutions to the micropolar fluid equations in exterior domains. Villamizar-Roa and Rodríguez-Bellido [29] studied the micropolar system in a bounded domain using the semigroup approach in $L^{p}$, showing the global existence of strong solutions for small data and the asymptotic behavior and stability of the solutions. Concerning the dynamical behavior of solutions to equations (1.1) one may refer to [4, 5, 7] and the references therein.

The purpose of this paper is to study the regularity of weak solutions and breakdown criteria for smooth solutions to the micropolar fluid equations (1.1). The classic blow-up criteria for smooth solutions to the Navier-Stokes equations also hold for the micropolar fluid equations. For the Navier-Stokes equations, Serrin 25], Prodi 23] and Veiga 28] established classic Serrin-type regularity criteria for weak solutions in terms of $u$ or its gradient $\nabla u$. Later, many improvements and extensions were established; for example, see [15, 13, 14, 32] and the references therein. Berselli and Galdi [2] and Chae and Lee [3] obtained regularity criteria for weak solutions in terms of the pressure $P$ or its gradient $\nabla P$. Later, Zhou extended the criteria in terms of the pressure and its gradient to a general domain [33, and Zhou [34, 35] and Struwe [27] obtained regularity criteria for weak solutions in terms of the gradient of pressure.

Theorem 1.1. Suppose $u \in L^{\infty}\left([0, T] ; L^{2}\left(\mathbb{R}^{n}\right)\right) \cap L^{2}\left([0, T] ; H^{1}\left(\mathbb{R}^{n}\right)\right)$ is a LerayHopf weak solution to the Navier-Stokes equations, and $P$ is the pressure. Suppose one of the following conditions is satisfied:

(1) $u \in L^{q}\left(0, T ; L^{p}\left(\mathbb{R}^{n}\right)\right)$ for $\frac{2}{q}+\frac{n}{p} \leq 1$ with $n<p \leq \infty$;

(2) $\nabla u \in L^{q}\left(0, T ; L^{p}\left(\mathbb{R}^{n}\right)\right)$ for $\frac{2}{q}+\frac{n}{p} \leq 2$ with $\frac{n}{2}<p \leq \infty$;

(3) $P \in L^{q}\left(0, T ; L^{p}\left(\mathbb{R}^{n}\right)\right)$ for $\frac{2}{q}+\frac{n}{p} \leq 2$ with $\frac{n}{2}<p \leq \infty$;

(4) $\nabla P \in L^{q}\left(0, T ; L^{p}\left(\mathbb{R}^{n}\right)\right)$ for $\frac{2}{q}+\frac{n}{p} \leq 3$ with $\frac{n}{3}<p<\infty$.

Then $u$ is a smooth solution on $[0, T)$.

Yuan [31] established regularity criteria, similar to the Serrin-type criteria, for weak solutions to the magneto-micropolar equations, which consist of the microploar equations (1.1) coupled with a magnetic field $b$, as follows.

Theorem 1.2. Let $\left(u_{0}, \omega_{0}, b_{0}\right) \in L^{2}\left(\mathbb{R}^{3}\right)$ with $\operatorname{div} u_{0}=\operatorname{div} b_{0}=0$. Assume that $(u, \omega, b)$ is a Leray-Hopf type weak solution to the magneto-micropolar equations. Suppose one of the following conditions holds:

(1) $u \in L^{q}\left(0, T ; L^{p}\left(\mathbb{R}^{3}\right)\right)$ for $\frac{2}{q}+\frac{3}{p} \leq 1$ with $3<p \leq \infty$;

(2) $\nabla u \in L^{q}\left(0, T ; L^{p}\left(\mathbb{R}^{3}\right)\right)$ for $\frac{2}{q}+\frac{3}{p} \leq 2$ with $\frac{3}{2}<p \leq \infty$.

Then $(u, \omega, b)$ is a smooth solution on $[0, T)$ with initial value $\left(u_{0}, \omega_{0}, b_{0}\right)$. 
It is worth noting that the regularity conditions for weak solutions to the magneto-micropolar equations are imposed only on the velocity field $u$, which is very important. For the magneto-hydrodynamic equations, He and Xin [12] first studied and established regularity criteria imposed only on the velocity field $u$ or its gradient $\nabla u$. Later, Zhou [36] improved these regularity criteria imposed only on $u$ or its gradient $\nabla u$, and He and Wang [11] improved them to weak $L^{p}$ spaces, imposed only on $u$ or its gradient $\nabla u$; Chen, Miao and Zhang [6] also improved the criteria to a more general Besov-type space on Littlewood-Paley decomposition imposed only on $\nabla \times u$. Regularity criteria for weak solutions to the system (1.1) play an important role in understanding the physical essence of micropolar fluid motion. The aim of this paper is to prove that to secure the regularity of weak solutions to (1.1), one only needs to impose conditions on the velocity field $u$, its gradient $\nabla u$ or the pressure of the fluid in Lorentz spaces. In particular, one needs only one of the following conditions to prove regularity of weak solutions $(u, \omega)$ on $[0, T]$ :

(1) $u \in L^{q}\left(0, T ; L^{p, \infty}\left(\mathbb{R}^{3}\right)\right)$ for $\frac{2}{q}+\frac{3}{p} \leq 1$ with $3<p \leq \infty$;

(2) $\nabla u \in L^{q}\left(0, T ; L^{p, \infty}\left(\mathbb{R}^{3}\right)\right)$ for $\frac{2}{q}+\frac{3}{p} \leq 2$ with $\frac{3}{2}<p \leq \infty$;

(3) $P \in L^{q}\left(0, T ; L^{p, \infty}\left(\mathbb{R}^{3}\right)\right)$ for $\frac{2}{q}+\frac{3}{p} \leq 2$ with $\frac{3}{2}<p \leq \infty$;

(4) $\nabla P \in L^{q}\left(0, T ; L^{p, \infty}\left(\mathbb{R}^{3}\right)\right)$ for $\frac{2}{q}+\frac{3}{p} \leq 3$ with $1<p<\infty$.

This demonstrates that, in the regularity of weak solutions, the micro-rotational velocity $\omega$ of particles plays a less important role than the velocity $u$ does, and the regularity of weak solutions to (1.1) is dominated by the velocity $u$ of the fluid.

We conclude this introduction by describing the plan of the paper. We give our main results on blow-up criteria for a smooth solution to (1.1) in section 2, and as an application we prove the regularity of weak solutions. Section 3 is devoted to proving Theorems 2.1 and 2.2 .

\section{MAin Results}

Before stating our main results we introduce some function spaces, notation and a generalized Hölder inequality. $C_{0, \sigma}^{\infty}\left(\mathbb{R}^{3}\right)$ denotes the set of all $C^{\infty}$ vector functions $f(x)=\left(f_{1}(x), f_{2}(x), f_{3}(x)\right)$ with compact support such that $\operatorname{div} f(x)=0 . L_{\sigma}^{r}\left(\mathbb{R}^{3}\right)$ is the closure of the $C_{0, \sigma}^{\infty}\left(\mathbb{R}^{3}\right)$-functions with respect to the $L^{r}$-norm $\|\cdot\|_{r}$ for $1 \leq r \leq \infty . H_{\sigma}^{s}\left(\mathbb{R}^{3}\right)$ denotes the closure of $C_{0, \sigma}^{\infty}\left(\mathbb{R}^{3}\right)$ with respect to the $H^{s}$-norm $\|f\|_{H^{s}}=\left\|(1-\Delta)^{\frac{s}{2}} f\right\|_{2}$, for $s \geq 0$.

In the following we recall Lorentz spaces. Let $(X, \mathcal{M}, \mu)$ be a non-atomic measurable space. For a complex- or real-valued $\mu$-measurable function $f(x)$ defined on $X$, its distribution function is defined by

$$
f_{*}(\sigma)=\mu\{x \in X:|f(x)|>\sigma\}, \text { for } \sigma>0,
$$

which is non-increasing and continuous from the right. Furthermore, its nonincreasing rearrangement $f^{*}$ is defined by

$$
f^{*}(t)=\inf \left\{s>0: f_{*}(s) \leq t\right\}, \text { for } t>0,
$$

which is also non-increasing and continuous from the right and has the same distribution function as $f(x)$. 
The Lorentz space $L^{p, q}$ on $(X, \mathcal{M}, \mu)$ is the collection of all real- or complexvalued $\mu$-measurable functions $f(x)$ defined on $X$ such that $\|f\|_{p, q}<\infty$, where

$$
\|f\|_{p, q}= \begin{cases}\left(\frac{q}{p} \int_{0}^{\infty}\left(t^{\frac{1}{p}} f^{*}(t)\right)^{q} \frac{\mathrm{d} t}{t}\right)^{\frac{1}{q}}, & \text { if } 1 \leq p<\infty, 1<q<\infty \\ \sup _{t>0} t^{\frac{1}{p}} f^{*}(t), & \text { if } 1 \leq p \leq \infty, q=\infty\end{cases}
$$

If $q=\infty$, we write $L^{p, \infty}\left(\mathbb{R}^{3}\right)$ as $L_{w}^{p}\left(\mathbb{R}^{3}\right)$, which is the weak $L^{p}$ space. Moreover

$$
\|f\|_{p, \infty}=\sup _{t>0} t^{\frac{1}{p}} f^{*}(t)=\sup _{\alpha>0} \alpha\left(f_{*}(\alpha)\right)^{\frac{1}{p}},
$$

for any $f(x) \in L^{p, \infty}$. For details see [20, 21] and [26].

We also need the Hölder inequality in Lorentz spaces, which we recall as follows; for details see O'Neil 22.

Proposition 2.1. Let $1<p_{1}, p_{2}, r<\infty$ with

$$
\frac{1}{p_{1}}+\frac{1}{p_{2}}<1, \quad \frac{1}{r}=\frac{1}{p_{1}}+\frac{1}{p_{2}}
$$

and $1 \leq q_{1}, q_{2}, s \leq \infty$ with

$$
\frac{1}{q_{1}}+\frac{1}{q_{2}} \geq \frac{1}{s}
$$

If $f \in L^{p_{1}, q_{1}}$ and $g \in L^{p_{2}, q_{2}}$, then $f g \in L^{r, s}$, and the generalized Hölder inequality

$$
\|h\|_{r, s} \leq r^{\prime}\|f\|_{p_{1}, q_{1}}\|g\|_{p_{2}, q_{2}}
$$

holds, where $r^{\prime}$ stands for the dual of $r$, i.e. $\frac{1}{r}+\frac{1}{r^{\prime}}=1$.

We state the main results as follows.

Theorem 2.1. Let $u_{0}(x) \in H_{\sigma}^{1}\left(\mathbb{R}^{3}\right)$ and $\omega_{0}(x) \in H^{1}\left(\mathbb{R}^{3}\right)$. Assume that the pair $u(t, x) \in C\left([0, T) ; H_{\sigma}^{1}\left(\mathbb{R}^{3}\right)\right) \cap C\left((0, T) ; H_{\sigma}^{2}\left(\mathbb{R}^{3}\right)\right)$ and $\omega(t, x) \in C\left([0, T) ; H^{1}\left(\mathbb{R}^{3}\right)\right) \cap$ $C\left((0, T) ; H^{2}\left(\mathbb{R}^{3}\right)\right)$ is a smooth solution to the equations (1.1). Suppose $u$ satisfies one of the following conditions:

(a) $u(t, x) \in L^{q}\left((0, T) ; L^{p, \infty}\left(\mathbb{R}^{3}\right)\right)$, for $\frac{2}{q}+\frac{3}{p} \leq 1$ with $3<p \leq \infty$;

(b) $\nabla u(t, x) \in L^{q}\left((0, T) ; L^{p, \infty}\left(\mathbb{R}^{3}\right)\right)$, for $\frac{2}{q}+\frac{3}{p} \leq 2$ with $\frac{3}{2}<p \leq \infty$.

Then the solution $(u, \omega)$ can be extended smoothly to $\left[0, T^{\prime}\right)$ for some $T^{\prime}>T$.

Theorem 2.2. Let $u_{0}(x) \in L_{\sigma}^{4}\left(\mathbb{R}^{3}\right)$ and $\omega_{0}(x) \in L^{4}\left(\mathbb{R}^{3}\right)$. Assume that the pair $u(t, x) \in C\left([0, T) ; L_{\sigma}^{4}\left(\mathbb{R}^{3}\right)\right) \cap C\left((0, T) ; H_{\sigma}^{1,4}\left(\mathbb{R}^{3}\right)\right)$ and $\omega(t, x) \in C\left([0, T) ; L^{4}\left(\mathbb{R}^{3}\right)\right) \cap$ $C\left((0, T) ; H^{1,4}\left(\mathbb{R}^{3}\right)\right)$ is a smooth solution to the equations (1.1), and $P$ is the pressure. If $P$ satisfies the condition

(1) $P(t, x) \in L^{q}\left((0, T) ; L^{p, \infty}\left(\mathbb{R}^{3}\right)\right)$, for $\frac{2}{q}+\frac{3}{p} \leq 2$ with $\frac{3}{2}<p \leq \infty$, or the gradient of the pressure $\nabla P$ satisfies the condition

(2) $\nabla P(t, x) \in L^{q}\left((0, T) ; L^{p, \infty}\left(\mathbb{R}^{3}\right)\right)$, for $\frac{2}{q}+\frac{3}{p} \leq 3$ with $1<p \leq \infty$, then the solution $(u, \omega)$ can be extended smoothly beyond $t=T$.

We next consider criteria for regularity of weak solutions to the micropolar equations (1.1); for this purpose we first introduce the definition of a weak solution. 
Definition 2.1. Let $u_{0}(x) \in L_{\sigma}^{2}\left(\mathbb{R}^{3}\right)$ and $\omega_{0}(x) \in L^{2}\left(\mathbb{R}^{3}\right)$. A measurable function $(u(t, x), \omega(t, x))$ is called a weak solution to the micropolar equations (1.1) on $[0, T)$ if

(a)

$$
\begin{aligned}
& u(t, x) \in L^{\infty}\left([0, T) ; L_{\sigma}^{2}\left(\mathbb{R}^{3}\right)\right) \cap L^{2}\left([0, T) ; H_{\sigma}^{1}\left(\mathbb{R}^{3}\right)\right), \\
& \omega(t, x) \in L^{\infty}\left([0, T) ; L^{2}\left(\mathbb{R}^{3}\right)\right) \cap L^{2}\left([0, T) ; H^{1}\left(\mathbb{R}^{3}\right)\right) ;
\end{aligned}
$$

(b)

$$
\begin{gathered}
\int_{0}^{T}\left\{-\left(u, \partial_{\tau} \varphi\right)+(\mu+\chi)(\nabla u, \nabla \varphi)-(u \cdot \nabla \varphi, u)+\chi(\nabla \times \varphi, \omega)\right\} \mathrm{d} \tau=\left(u_{0}, \varphi(0)\right), \\
\int_{0}^{T}\left\{\begin{array}{c}
-\left(\omega, \partial_{\tau} \phi\right)+\gamma(\nabla \omega, \nabla \phi)+\kappa(\operatorname{div} \omega, \operatorname{div} \phi) \\
+2 \chi(\omega, \phi)-(u \cdot \nabla \phi, \omega)+\chi(\nabla \times \phi, u)\} \mathrm{d} \tau=\left(\omega_{0}, \phi(0)\right),
\end{array}\right.
\end{gathered}
$$

for any $\varphi(t, x) \in H^{1}\left([0, T) ; H_{\sigma}^{1}\left(\mathbb{R}^{3}\right)\right)$ and $\phi(t, x) \in H^{1}\left([0, T) ; H^{1}\left(\mathbb{R}^{3}\right)\right)$ with $\varphi(T)=$ 0 and $\phi(T)=0$.

In 24, Rojas-Medar and Boldrini proved the global existence of weak solutions to the equations of magneto-micropolar fluid motion by the Galerkin method. The weak solutions $(u, \omega)$ also satisfy the energy inequality

$$
\begin{aligned}
& \|(u, \omega)\|_{2}^{2}+2 \mu \int_{0}^{t}\|\nabla u\|_{2}^{2} \mathrm{~d} s+2 \gamma \int_{0}^{t}\|\nabla \omega\|_{2}^{2} \mathrm{~d} s+2 \kappa \int_{0}^{t}\|\operatorname{div} \omega\|_{2}^{2} \mathrm{~d} s+2 \chi \int_{0}^{t}\|\omega\|_{2}^{2} \mathrm{~d} s \\
& \quad \leq\|(u(0), \omega(0))\|_{2}^{2},
\end{aligned}
$$

for $0<t \leq T$.

As immediate corollaries we establish the regularity criteria for weak solutions.

Corollary 2.1. Let $u_{0}(x) \in H_{\sigma}^{1}\left(\mathbb{R}^{3}\right)$ and $\omega_{0}(x) \in H^{1}\left(\mathbb{R}^{3}\right)$. Assume $(u(t, x), \omega(t, x))$ is a weak solution to the equations (1.1) and satisfies the energy inequality (2.6). Suppose $u$ satisfies one of the following conditions:

(a) $u(t, x) \in L^{q}\left((0, T) ; L^{p, \infty}\left(\mathbb{R}^{3}\right)\right)$ for $\frac{2}{q}+\frac{3}{p} \leq 1$ with $3<p \leq \infty$;

(b) $\nabla u(t, x) \in L^{q}\left((0, T) ; L^{p, \infty}\left(\mathbb{R}^{3}\right)\right)$ for $\frac{2}{q}+\frac{3}{p} \leq 2$ with $\frac{3}{2}<p \leq \infty$.

Then the solution $(u, \omega)$ is a regular solution on $(0, T]$.

Corollary 2.2. Let $u_{0}(x) \in L_{\sigma}^{2} \cap L_{\sigma}^{4}\left(\mathbb{R}^{3}\right)$ and $\omega_{0}(x) \in L^{2} \cap L^{4}\left(\mathbb{R}^{3}\right)$. Assume that $(u(t, x), \omega(t, x))$ is a weak solution to the equations (1.1) and satisfies the energy inequality (2.6), and $P$ is the pressure. If $P$ satisfies the condition

(1) $P(t, x) \in L^{q}\left((0, T) ; L^{p, \infty}\left(\mathbb{R}^{3}\right)\right)$, for $\frac{2}{q}+\frac{3}{p} \leq 2$ with $\frac{3}{2}<p \leq \infty$, or the gradient of the pressure $\nabla P$ satisfies the condition

(2) $\nabla P(t, x) \in L^{q}\left((0, T) ; L^{p, \infty}\left(\mathbb{R}^{3}\right)\right)$, for $\frac{2}{q}+\frac{3}{p} \leq 3$ with $1<p \leq \infty$, then the solution $(u, \omega)$ is a regular solution on $(0, T]$.

Remark 2.1. In Theorems 2.1 and 2.2, if $p=\infty$, then the space $L^{\infty, \infty}$ is identified with $L^{\infty}$.

Remark 2.2. In this paper the regularity results Theorem 2.1 and Corollary 2.1 are established for the solution of the micropolar equations (1.1). By the coupling of the velocity field and the magnetic field, the conclusions of Theorem 2.1 and Corollary 2.1 are also valid for the magneto-micropolar equations. 
Remark 2.3. For the magneto-hydrodynamic equations, He and Wang [11] proved that to assure the regularity of weak solutions one needs only the condition

$$
\nabla u \in L^{q}\left((0, T) ; L^{p, \infty}\left(\mathbb{R}^{3}\right)\right),
$$

for $\frac{2}{q}+\frac{3}{p}=2$ with $1<q \leq 2$. In our Theorem 2.1(b) and Corollary 2.2(b) the condition $1 \leq q<\infty$ is more general than that for (2.7). Moreover, our proof, which is based on a priori estimate of the $H^{1}$-norm of the solution, is simple, while that in 11 was based on a priori estimate of the $L^{p}$-norm for $p \geq 3$.

The proofs of Corollaries 2.1 and 2.2 are standard. For completeness we sketch the proof of Corollary 2.1 only. Since $u_{0}(x) \in H_{\sigma}^{1}\left(\mathbb{R}^{3}\right)$ and $\omega_{0}(x) \in H^{1}\left(\mathbb{R}^{3}\right)$, by the local existence theorem for strong solutions to the micropolar equations (1.1) there exists a unique solution $(\hat{u}, \hat{\omega})$ with $\hat{u}(t, x) \in C\left(\left[0, T^{*}\right) ; H_{\sigma}^{1}\left(\mathbb{R}^{3}\right)\right)$ and $\hat{\omega}(t, x) \in C\left(\left[0, T^{*}\right) ; H^{1}\left(\mathbb{R}^{3}\right)\right)$ on a small time interval $\left[0, T^{*}\right)$. Since $(u, \omega)$ is a weak solution satisfying the energy inequality (2.6), it follows from the Serrintype uniqueness criterion [25] that $(u(t), \omega(t)) \equiv(\hat{u}(t), \hat{\omega}(t))$ on $\left[0, T^{*}\right)$. Thus it is sufficient to show that $T=T^{*}$. If not, suppose that $T^{*}<T$. Without loss of generality, one may assume that $T^{*}$ is the maximal existence time of the strong solution $(\hat{u}, \hat{\omega})$. By condition (a) or (b) in Corollary 2.1 we have

$$
\int_{0}^{T^{*}}\|\hat{u}(t)\|_{p, \infty}^{q} \mathrm{~d} t<\infty, \text { for } \frac{2}{q}+\frac{3}{p} \leq 1 \text { with } 3<p \leq \infty
$$

or

$$
\int_{0}^{T^{*}}\|\nabla \hat{u}(t)\|_{p, \infty}^{q} \mathrm{~d} t<\infty, \text { for } \frac{2}{q}+\frac{3}{p} \leq 2 \text { with } \frac{3}{2}<p \leq \infty
$$

because $(\hat{u}(t), \hat{\omega}(t)) \equiv(u(t), \omega(t))$. Therefore it follows from Theorem 2.1 that there exists a time $T^{\prime}>T^{*}$ such that $(\hat{u}, \hat{\omega})$ can be extended smoothly to $\left[0, T^{\prime}\right)$, which contradicts the maximality of $T^{*}$. We thus complete the proof of Corollary 2.1.

In the following arguments the letter $C$ denotes an inessential constant which may vary from line to line but does not depend on particular solutions or functions. We also use $C(\chi, \gamma, \cdots)$ to denote a constant which depends on the parameters $\chi, \gamma, \cdots$ and may vary from line to line.

\section{Proof of Theorems 2.1 and 2.2}

In this section we prove Theorems 2.1 and 2.2 by a simple method.

Proof of Theorem 2.1. We differentiate the equations (1.1) with respect to $x_{i}$, then multiply the resulting equations by $\partial_{x_{i}} u$ and $\partial_{x_{i}} \omega$ for $i=1,2,3$, integrate with respect to $x$ and sum them up. It follows that

$$
\begin{aligned}
& \frac{1}{2} \frac{\mathrm{d}}{\mathrm{d} t}\left(\left\|\left(\partial_{x_{i}} u, \partial_{x_{i}} \omega\right)\right\|_{2}^{2}\right)+\sum_{j=1}^{3}\left((\mu+\chi)\left\|\partial_{x_{i} x_{j}}^{2} u\right\|_{2}^{2}+\gamma\left\|\partial_{x_{i} x_{j}}^{2} \omega\right\|_{2}^{2}\right) \\
& \quad+\kappa\left\|\operatorname{div} \partial_{x_{i}} \omega\right\|_{2}^{2}+2 \chi\left\|\partial_{x_{i}} \omega\right\|_{2}^{2} \\
& \leq\left|\left(\partial_{x_{i}} u \cdot \nabla u, \partial_{x_{i}} u\right)\right|+\left|\left(\partial_{x_{i}} u \cdot \nabla \omega, \partial_{x_{i}} \omega\right)\right|+2 \chi\left|\left(\nabla \times \partial_{x_{i}} u, \partial_{x_{i}} \omega\right)\right| \\
& =I_{1}+I_{2}+I_{3},
\end{aligned}
$$

where we have used the facts that

$$
\left(\nabla \times \partial_{x_{i}} u, \partial_{x_{i}} \omega\right)=\left(\nabla \times \partial_{x_{i}} \omega, \partial_{x_{i}} u\right)
$$


and

$$
\left(u \cdot \nabla \partial_{x_{i}} u, \partial_{x_{i}} u\right)=\left(u \cdot \nabla \partial_{x_{i}} \omega, \partial_{x_{i}} \omega\right)=0,
$$

where $(\cdot, \cdot)$ denotes the $L^{2}$ inner product on $\mathbb{R}^{3}$. For conciseness, the short notation

$$
\|(A, B)\|_{2}^{2}=\|A\|_{2}^{2}+\|B\|_{2}^{2}
$$

has been used and will be used in the following arguments.

(a) We estimate the terms $I_{1}, I_{2}$ and $I_{3}$. After integrations by parts and using the generalized Hölder inequality (2.5) it follows that

$$
\begin{aligned}
I_{1} & \leq\left|\int_{\mathbb{R}^{3}} \partial_{x_{i}} u \cdot \nabla \partial_{x_{i}} u \cdot u(x) \mathrm{d} x\right|+\left|\int_{\mathbb{R}^{3}} \partial_{x_{i}} \partial_{x_{i}} u \cdot \nabla u \cdot u(x) \mathrm{d} x\right| \\
& \leq C(p)\|u\|_{p, \infty}\left(\left\|\nabla \partial_{x_{i}} u \partial_{x_{i}} u\right\|_{\frac{p}{p-1}, 1}+\left\|\partial_{x_{i} x_{i}} u \partial_{x_{i}} u\right\|_{\frac{p}{p-1}, 1}\right) \\
& \leq C(p)\|u\|_{p, \infty}\|\nabla u\|_{\frac{2 p}{p-2}, 2}\left\|D^{2} u\right\|_{2} .
\end{aligned}
$$

Applying the real interpolation (see [1])

$$
L^{\frac{2 p}{p-2}, 2}\left(\mathbb{R}^{3}\right)=\left(L^{2}, L^{6}\right)_{\frac{p-3}{p}, 2}\left(\mathbb{R}^{3}\right)
$$

and the Sobolev embedding $L^{6}\left(\mathbb{R}^{3}\right) \hookrightarrow \dot{H}^{1}\left(\mathbb{R}^{3}\right)$ yields that

$$
\|\nabla u\|_{\frac{2 p}{p-2}, 2} \leq C\|\nabla u\|_{2}^{1-\frac{3}{p}}\left\|D^{2} u\right\|_{2}^{\frac{3}{p}} .
$$

Inserting the above estimate (3.6) into the estimate (3.5) for $I_{1}$ one has

$$
\begin{aligned}
I_{1} & \leq C(p)\|u\|_{p, \infty}\|\nabla u\|_{2}^{1-\frac{3}{p}}\left\|D^{2} u\right\|^{1+\frac{3}{p}} \\
& \leq \frac{\chi}{12}\left\|D^{2} u\right\|^{2}+C(p, \chi)\|u\|_{p, \infty}^{\frac{2 p}{p-3}}\|\nabla u\|_{2}^{2} .
\end{aligned}
$$

Similarly, for $I_{2}$ one also has

$$
I_{2} \leq \frac{\gamma}{6}\left\|D^{2} \omega\right\|^{2}+C(p, \gamma)\|u\|_{p, \infty}^{\frac{2 p}{p-3}}\|\nabla \omega\|_{2}^{2}
$$

For the term $I_{3}$, the Hölder and Young inequalities imply that

$$
I_{3} \leq \frac{\chi}{2}\left\|\nabla \times \partial_{x_{i}} u\right\|_{2}^{2}+2 \chi\|\nabla \omega\|_{2}^{2}
$$

Inserting the estimates (3.7)-(3.9) into the inequality (3.1) and summing over $i$ from 1 to 3 , it follows that

$$
\begin{aligned}
& \frac{\mathrm{d}}{\mathrm{d} t}\left(\|(\nabla u, \nabla \omega)\|_{2}^{2}\right)+\left(2 \mu+\frac{1}{2} \chi\right)\left\|D^{2} u\right\|_{2}^{2}+\gamma\left\|D^{2} \omega\right\|_{2}^{2}+2 \kappa\|\nabla \operatorname{div} \omega\|_{2}^{2} \\
& \quad \leq C(p, \chi, \gamma)\|u\|_{p, \infty}^{2 p /(p-3)}\|(\nabla u, \nabla \omega)\|_{2}^{2} .
\end{aligned}
$$

Gronwall's inequality leads to the a priori estimate

$$
\|(\nabla u, \nabla \omega)\|_{2}^{2} \leq\left\|\left(\nabla u_{0}, \nabla \omega_{0}\right)\right\|_{2}^{2} \exp \left\{C(p, \chi, \gamma) \int_{0}^{t}\|u(s)\|_{p, \infty}^{2 p /(p-3)} \mathrm{d} s\right\} .
$$


In case (b), we estimate $I_{1}$ and $I_{2}$ in another way. Using the generalized Hölder's and Young's inequalities, we have

$$
\begin{aligned}
I_{1} & \leq C\|\nabla u\|_{p, \infty}\|\nabla u\|_{\frac{p}{p-1}, 1} \leq C(p)\|\nabla u\|_{p, \infty}\|\nabla u\|_{\frac{2 p}{p-1}, 2}^{2} \\
& \leq C(p)\|\nabla u\|_{p, \infty}\|\nabla u\|_{2}^{2-\frac{3}{p}}\left\|D^{2} u\right\|_{2}^{\frac{3}{p}} \\
& \leq \frac{\chi}{12}\left\|D^{2} u\right\|_{2}^{2}+C(p, \chi)\|\nabla u\|_{p, \infty}^{\frac{2 p}{2 p-3}}\|\nabla u\|_{2}^{2},
\end{aligned}
$$

where use has been made of the facts

$$
L^{\frac{2 p}{p-1}, 2}\left(\mathbb{R}^{3}\right)=\left(L^{2}, L^{6}\right)_{\frac{2 p-3}{2 p}, 2}\left(\mathbb{R}^{3}\right)
$$

and

$$
\|\nabla u\|_{\frac{2 p}{p-2}, 2}^{2} \leq C\|\nabla u\|_{2}^{2-\frac{3}{p}}\left\|D^{2} u\right\|_{2}^{\frac{3}{p}}
$$

Arguing similarly, $I_{2}$ can be estimated as follows:

$$
I_{2} \leq \frac{\gamma}{6}\left\|D^{2} \omega\right\|^{2}+C(p, \gamma)\|\nabla u\|_{p, \infty}^{\frac{2 p}{2 p-3}}\|\nabla \omega\|_{2}^{2}
$$

Inserting the estimates (3.11), (3.12) and (3.9) into (3.1), summing over $i$ from 1 to 3, and applying Gronwall's inequality give the a priori estimate

$$
\|(\nabla u, \nabla \omega)\|_{2}^{2} \leq\left\|\left(\nabla u_{0}, \nabla \omega_{0}\right)\right\|_{2}^{2} \exp \left\{C(p, \chi, \gamma) \int_{0}^{t}\|\nabla u(s)\|_{p, \infty}^{2 p /(2 p-3)} \mathrm{d} s\right\} .
$$

The above estimates are also valid for $p=\infty$ provided we modify them accordingly.

Combining the a priori estimates (3.10) and (3.13) with the energy inequality (2.6) and using standard arguments for continuation of local solutions, we conclude that the solution $(u(t, x), \omega(t, x))$ can be extended beyond $t=T$ provided that $u(t, x) \in L^{q}\left(0, T ; L^{p, \infty}\left(\mathbb{R}^{3}\right)\right)$ for $\frac{2}{q}+\frac{3}{p} \leq 1$ with $3<p \leq \infty$, or $\nabla u(t, x) \in L^{q}\left((0, T) ; L^{p, \infty}\left(\mathbb{R}^{3}\right)\right)$ for $\frac{2}{q}+\frac{3}{p} \leq 2$ with $\frac{3}{2}<p \leq \infty$. The proof of Theorem 2.1 is thus complete.

Proof of Theorem 2.2. To prove the theorem we need an $L^{4}$ a priori estimate. For this purpose, we take the inner product of the first equation of (1.1) with $|u|^{2} u$ and integrate by parts; it can be deduced that

$$
\begin{aligned}
& \frac{1}{4} \frac{\mathrm{d}}{\mathrm{d} t}\|u\|_{4}^{4}+(\mu+\chi) \int_{\mathbb{R}^{3}}|\nabla u|^{2}|u|^{2} \mathrm{~d} x+\left.\left.\frac{1}{2}(\mu+\chi) \int_{\mathbb{R}^{3}}|\nabla| u\right|^{2}\right|^{2} \mathrm{~d} x \\
& \leq 2 \int_{\mathbb{R}^{3}}|P \| u|^{2}|\nabla u| \mathrm{d} x+3 \chi \int_{\mathbb{R}^{3}}|w||u|^{2}|\nabla u| \mathrm{d} x,
\end{aligned}
$$

where we used the following relations arising from the divergence free condition $\operatorname{div} u=0$ :

$$
\begin{aligned}
& \int_{\mathbb{R}^{3}} u \cdot \nabla u \cdot|u|^{2} u \mathrm{~d} x=\frac{1}{2} \int_{\mathbb{R}^{3}} u \cdot \nabla|u|^{4} \mathrm{~d} x=0, \\
& \int_{\mathbb{R}^{3}} \Delta u \cdot|u|^{2} u \mathrm{~d} x=-\int_{\mathbb{R}^{3}}|\nabla u|^{2}|u|^{2} \mathrm{~d} x-\left.\left.\frac{1}{2} \int_{\mathbb{R}^{3}}|\nabla| u\right|^{2}\right|^{2} \mathrm{~d} x, \\
& \int_{\mathbb{R}^{3}} \nabla \times \omega \cdot|u|^{2} u \mathrm{~d} x=-\int_{\mathbb{R}^{3}}|u|^{2} \omega \cdot \nabla \times u \mathrm{~d} x-\int_{\mathbb{R}^{3}} \omega \cdot \nabla|u|^{2} \times u \mathrm{~d} x,
\end{aligned}
$$

and

$$
|\nabla \times u| \leq|\nabla u|,|\nabla| u|| \leq|\nabla u| .
$$


Using an argument similar to that used in deriving the estimate (3.14) it can be obtained for the second equation of (1.1) that

$$
\begin{aligned}
\frac{1}{4} \frac{\mathrm{d}}{\mathrm{d} t}\|\omega\|_{4}^{4} & +\gamma \int_{\mathbb{R}^{3}}|\nabla \omega|^{2}|\omega|^{2} \mathrm{~d} x+\left.\left.\frac{1}{2} \gamma \int_{\mathbb{R}^{3}}|\nabla| \omega\right|^{2}\right|^{2} \mathrm{~d} x \\
& +\left.\kappa \int_{\mathbb{R}^{3}} \operatorname{div} \omega\right|^{2} \mathrm{~d} x+2 \chi \int_{\mathbb{R}^{3}}|\omega|^{4} \mathrm{~d} x \\
\leq & 3 \chi \int_{\mathbb{R}^{3}}|u||\omega|^{2}|\nabla \omega| \mathrm{d} x .
\end{aligned}
$$

Combining estimates (3.14) and (3.15), we arrive at

$$
\begin{aligned}
& \frac{1}{4} \frac{\mathrm{d}}{\mathrm{d} t}\left(\|u\|_{4}^{4}+\|\omega\|_{4}^{4}\right)+(\mu+\chi) \int_{\mathbb{R}^{3}}|\nabla u|^{2}|u|^{2} \mathrm{~d} x+\left.\left.\frac{1}{2}(\mu+\chi) \int_{\mathbb{R}^{3}}|\nabla| u\right|^{2}\right|^{2} \mathrm{~d} x \\
& +\gamma \int_{\mathbb{R}^{3}}|\nabla \omega|^{2}|\omega|^{2} \mathrm{~d} x+\left.\left.\frac{1}{2} \gamma \int_{\mathbb{R}^{3}}|\nabla| \omega\right|^{2}\right|^{2} \mathrm{~d} x+\kappa \int_{\mathbb{R}^{3}}|\operatorname{div} \omega|^{2} \mathrm{~d} x+2 \chi \int_{\mathbb{R}^{3}}|\omega|^{4} \mathrm{~d} x \\
& \leq 2 \int_{\mathbb{R}^{3}}|P||u|^{2}|\nabla u| \mathrm{d} x+3 \chi \int_{\mathbb{R}^{3}}|w||u|^{2}|\nabla u| \mathrm{d} x+3 \chi \int_{\mathbb{R}^{3}}|u||\omega|^{2}|\nabla \omega| \mathrm{d} x \\
& =I+I I+I I I .
\end{aligned}
$$

Applying Hölder's and Young's inequalities for $I I$, it follows that

$$
I I \leq \frac{1}{2} \chi \int_{\mathbb{R}^{3}}|\nabla u|^{2}|u|^{2} \mathrm{~d} x+C(\chi)\left(\|u\|_{4}^{4}+\|\omega\|_{4}^{4}\right) .
$$

Arguing similarly to above we can derive for III that

$$
I I I \leq \frac{1}{2} \gamma \int_{\mathbb{R}^{3}}|\nabla \omega|^{2}|\omega|^{2} \mathrm{~d} x+C(\gamma)\left(\|u\|_{4}^{4}+\|\omega\|_{4}^{4}\right) .
$$

Concerning the term $I$, by virtue of the generalized Hölder's inequality (2.5) we have

$$
\begin{aligned}
& I \leq C(p)\left\||P|^{1 / 2}\right\|_{2 p, \infty}\left\||P|^{1 / 2}|u|\right\|_{\frac{2 p}{p-1}, 2}\|u \nabla u\|_{2} \\
& \leq C(p)\|P\|_{p, \infty}^{1 / 2}\|u \nabla u\|_{2}\|P\|_{\frac{2 p}{p-1}, 2}^{1 / 2}\|u\|_{\frac{4 p}{p-1}, 4} .
\end{aligned}
$$

Applying the divergence operator div to the first equation of (1.1), one formally has

$$
P=\sum_{i, j=1}^{3} R_{i} R_{j} u_{i} u_{j},
$$

where $R_{j}$ denotes the $j$-th Riesz operator. By the boundedness of the Riesz operator on a Lorentz space $L^{p, q}\left(\mathbb{R}^{3}\right)$ for $1<p \leq q<\infty$, and upon applying the generalized Hölder's inequality (2.5) again, we obtain that

$$
\|P\|_{\frac{2 p}{p-1}, 2} \leq C\|u\|_{\frac{4 p}{p-1}, 4}^{2} .
$$

So the term $I$ can be estimated as

$$
I \leq C(p)\|P\|_{p, \infty}^{1 / 2}\|u \nabla u\|_{2}\|u\|_{\frac{4 p}{p-1}, 4}^{2} .
$$

In view of the real interpolation

$$
L^{\frac{4 p}{p-1}, 4}\left(\mathbb{R}^{3}\right)=\left(L^{4}, L^{12}\right)_{\frac{2 p-3}{2 p}, 4}\left(\mathbb{R}^{3}\right)
$$


and the Sobolev's inequality we have

$$
\|u\|_{\frac{4 p}{p-1}, 4}^{2} \leq C\|u\|_{4}^{\frac{2 p-3}{p}}\|u\|_{12}^{\frac{3}{p}} \leq C\|u\|_{4}^{\frac{2 p-3}{p}}\|u \nabla|u|\|_{2}^{\frac{3}{2 p}} .
$$

Inserting the estimate (3.22) into the estimate (3.21) for $I$, one estimates $I$ as

$$
I \leq C(p)\|P\|_{p, \infty}^{1 / 2}\|u\|_{4}^{\frac{2 p-3}{p}}\|u \nabla u\|_{2}^{\frac{2 p+3}{2 p}} \leq C(p, \chi)\|P\|_{p, \infty}^{\frac{2 p}{2 p-3}}\|u\|_{4}^{4}+\frac{1}{2} \chi\|u \nabla u\|_{2}^{2} .
$$

Inserting the estimates (3.23), (3.17) and (3.18) for I, II and III into (3.16) we get that

$$
\frac{1}{4} \frac{\mathrm{d}}{\mathrm{d} t}\left(\|u\|_{4}^{4}+\|\omega\|_{4}^{4}\right) \leq C(p, \chi)\|P\|_{p, \infty}^{\frac{2 p}{22-3}}\|u\|_{4}^{4}+C(\chi, \gamma)\left(\|u\|_{4}^{4}+\|\omega\|_{4}^{4}\right) .
$$

Gronwall's inequality implies that

$$
\|u\|_{4}^{4}+\|\omega\|_{4}^{4} \leq\left(\left\|u_{0}\right\|_{4}^{4}+\left\|\omega_{0}\right\|_{4}^{4}\right) \exp \left\{C(p, \chi, \gamma) \int_{0}^{t}\left(1+\|P\|_{p, \infty}^{\frac{2 p}{2 p-3}}\right) \mathrm{d} s\right\} .
$$

In case (2), we estimate $I$ by another method. First, $I$ also equals $\int_{\mathbb{R}^{3}} \nabla P$. $u|u|^{2} \mathrm{~d} x$, so

$$
\begin{aligned}
I & \leq \int_{\mathbb{R}^{3}}|\nabla P|^{1 / 2}|\nabla P|^{1 / 2}|u|^{3} \mathrm{~d} x \\
& \leq C\left\||\nabla P|^{1 / 2}\right\|_{2 p, \infty}\left\||\nabla P|^{1 / 2}|u|^{3}\right\|_{\frac{2 p}{2 p-1}, 1} \\
& \leq C(p)\|\nabla P\|_{p, \infty}^{1 / 2}\|\nabla P\|_{2}^{1 / 2}\|u\|_{\frac{12 p}{3 p-2}, 4}^{3},
\end{aligned}
$$

where we have used the generalized Hölder's inequality (2.5). Noting the relation (3.19) between $P$ and $u$, the Riesz operator's boundedness on $L^{p}$ for $1<p<\infty$ and the real interpolation

$$
L^{\frac{12 p}{3 p-2}, 4}\left(\mathbb{R}^{3}\right)=\left(L^{4}, L^{12}\right) \frac{p-1}{p}, 4\left(\mathbb{R}^{3}\right),
$$

the term $I$ can be estimated as

$$
\begin{aligned}
I & \leq C(p)\|\nabla P\|_{p, \infty}^{1 / 2}\|u \nabla u\|_{2}^{1 / 2}\|u\|_{4}^{\frac{3(p-1)}{p}}\|u\|_{12}^{\frac{3}{p}} \\
& \leq C(p)\|\nabla P\|_{p, \infty}^{1 / 2}\|u \nabla u\|_{2}^{\frac{p+3}{2 p}}\|u\|_{4}^{\frac{3(p-1)}{p}} \\
& \leq C(p, \chi)\|\nabla P\|_{p, \infty}^{\frac{2}{3(p-1)}}\|u\|_{4}^{4}+\frac{1}{2} \chi\|u \nabla u\|_{2}^{2},
\end{aligned}
$$

where the Sobolev embedding $L^{6}\left(\mathbb{R}^{3}\right) \hookrightarrow \dot{H}^{1}\left(\mathbb{R}^{3}\right)$ and Young's inequality were used. Inserting the estimates (3.25), (3.17) and (3.18) for I, II and III into (3.16) one also has

$$
\frac{1}{4} \frac{\mathrm{d}}{\mathrm{d} t}\left(\|u\|_{4}^{4}+\|\omega\|_{4}^{4}\right) \leq C(p, \chi)\|\nabla P\|_{p, \infty}^{\frac{2 p}{3(p-1)}}\|u\|_{4}^{4}+C(\chi, \gamma)\left(\|u\|_{4}^{4}+\|\omega\|_{4}^{4}\right) .
$$

Apply Gronwall's inequality again to arrive at

$$
\|u\|_{4}^{4}+\|\omega\|_{4}^{4} \leq\left(\left\|u_{0}\right\|_{4}^{4}+\left\|\omega_{0}\right\|_{4}^{4}\right) \exp \left\{C(p, \chi, \gamma) \int_{0}^{t}\left(1+\|\nabla P\|_{p, \infty}^{\frac{2 p}{3(p-1)}}\right) \mathrm{d} s\right\} .
$$

The above estimates are also valid for $p=\infty$ provided we modify them accordingly.

Combining the a priori estimates (3.24) and (3.26) with the energy inequality (2.6) and using standard arguments for continuation of local solutions, we conclude that the solution $(u(t, x), \omega(t, x))$ can be extended beyond $t=T$ provided that 
$P(t, x) \in L^{q}\left((0, T) ; L^{p, \infty}\left(\mathbb{R}^{3}\right)\right)$ for $\frac{2}{q}+\frac{3}{p} \leq 2$ with $\frac{3}{2}<p \leq \infty$, or $\nabla P(t, x) \in$ $L^{q}\left((0, T) ; L^{p, \infty}\left(\mathbb{R}^{3}\right)\right)$ for $\frac{2}{q}+\frac{3}{p} \leq 3$ with $1<p \leq \infty$. We thus complete the proof of Theorem 2.2.

\section{REFERENCES}

1. Bergh, J., Löfström, J., Interpolation spaces: an introduction. Berlin-New York: SpringerVerlag, 1976. MR 0482275 (58:2349)

2. Berselli, L. C., Galdi, G. P., Regularity criteria involving the pressure for the weak solutions to the Navier-Stokes equations. Proc. Amer. Math. Soc., 2002, 130(12):3585-3595. MR.1920038 (2003e:35240)

3. Chae, D., Lee, J., Regularity criterion in terms of pressure for the Navier-Stokes equations. Nonlinear Analysis, 2001, 46:727-735. MR 1857154 (2002g:76032)

4. Chen, J. W., Dong, B. Q., Chen, Z. M., Pullback attractors of non-autonomous micropolar fluid flows. J. Math. Anal. Appl., 2007, 336:1384-1394. MR2353021 (2008g:37073)

5. Chen, J. W., Chen, Z. M., Dong, B. Q., Uniform attractors of non-homogeneous micropolar fluid flows in non-smooth domains. Nonlinearity, 2007, 20:1619-1635. MR2335076 (2008i:37158)

6. Chen, Q. L., Miao, C. X., Zhang, Z. F., The Beale-Kato-Majda criterion for the 3D magnetohydrodynamics equations. Comm. Math. Phys., 2007, 275:861-872. MR2336368|(2008i:76203)

7. Dong, B. Q., Chen, Z. M., On upper and lower bounds of higher order derivatives for solutions to the 2D micropolar fluid equations. J. Math. Anal. Appl., 2007, 334:1386-1399. MR2338669 (2008h:35285)

8. Eringen, A. C., Theory of micropolar fluids. J. Math. Mech., 1966, 16:1-18. MR0204005 $(34: 3852)$

9. Ferreira, L. C. F., Villamizar-Roa, E. J., On the existence and stability of solutions for the micropolar fluids in exterior domains. Math. Meth. Appl. Sci., 2007, 30:1185-1208. MR2329639 (2009d:35260)

10. Galdi, G. P., Rionero, S., A note on the existence and uniqueness of solutions of the micropolar fluid equations. Internat. J. Engrg. Sci., 1977, 15:105-108. MR0467030 (57:6899)

11. He, C., Wang, Y., On the regularity criteria for weak solutions to the magnetohydrodynamic equations. J. Diff. Eqs., 2007, 238:1-17. MR2334589 (2008e:35152)

12. He, C., Xin, Z. P., On the regularity of weak solutions to the magnetohydrodynamic equations. J. Diff. Eqs., 2005, 213:235-254. MR2142366 (2006f:35217)

13. Kozono, H., Ogawa, T., Taniuchi, Y., The critical Sobolev inequalities in Besov spaces and regularity criterion to some semi-linear evolution equations. Math. Z., 2002, 242:251-278. MR:1980623(2004c:35324)

14. Kozono, H., Shimada, Y., Bilinear estimates in homogeneous Triebel-Lizorkin spaces and the Navier-Stokes equations. Math. Nachr., 2004, 276:63-74. MR.2100048 (2006a:35236)

15. Kozono, H., Taniuchi, Y., Bilinear estimates in BMO and the Navier-Stokes equations. Math. Z., 2000, 235, 173-194. MR.1785078 (2001g:76011)

16. Ladyzhenskaya, O., The mathematical theory of viscous incompressible flow. New York: Gordon and Breach, 1969. MR0254401 (40:7610)

17. Lange, H., The existence of instationary flows of incompressible micropolar fluids. Arch. Mech., 1977, 29:741-744. MR0489300 (58:8741)

18. Lemarié-Rieusset, P. G., Recent developments in the Navier-Stokes problem. Boca Raton, FL: Chapman \& Hall/CRC, 2002. MR1938147(2004e:35178)

19. Lions, P. L., Mathematical topics in fluid mechanics. Vol. 1. New York: The Clarendon Press, Oxford University Press, 1996. MR.1422251 (98b:76001)

20. Lorentz, G. G., Some new functional spaces. Ann. of Math. (2), 1950, 51:37-55. MR0033449 $(11: 442 \mathrm{~d})$

21. Miao, C. X., Harmonic analysis and application to partial differential equations. 2nd ed., Beijing: Science Press, 2004.

22. O'Neil, R., Convolution operators and $L_{p, q}$ spaces. Duke Math. J., 1963, 30:129-142. MR0146673 (26:4193)

23. Prodi, G., Un teorema di unicità per le equazioni di Navier-Stokes. Ann. Mat. Pura Appl., 1959, 48(4):173-182. MR0126088 (23:A3384) 
24. Rojas-Medar, M. A., Boldrini, J. L., Magneto-micropolar fluid motion: existence of weak solutions. Rev. Mat. Complut., 1998, 11:443-460. MR1666509 (99k:76154)

25. Serrin, J., The initial value problem for the Navier-Stokes equations, Nonlinear problems (Proc. Sympos., Madison, WI). Madison: University of Wisconsin Press, 1963, 69-98. MR0150444 (27:442)

26. Stein, E. M., Weiss, G., Introduction to Fourier analysis on Euclidean spaces. Princeton, NJ: Princeton University Press, 1971. MR0304972 (46:4102)

27. Struwe, M., On a Serrin-type regularity criterion for the Navier-Stokes equations in terms of the pressure. J. Math. Fluid Mech., 2007, 9:235-242. MR2329267(2008i:35185)

28. Beirão da Veiga, H., A new regularity class for the Navier-Stokes equations in $\mathbb{R}^{n}$. Chinese Ann. Math. Ser. B, 1995, 16(4):407-412. MR.1380578 (96m:35035)

29. Villamizar-Roa, E. J., Rodríguez-Bellido, M. A., Global existence and exponential stability for the micropolar fluid system. Z. Angew. Math. Phys., 2008, 59:790-809. MR 2442951 (2009k:76006)

30. Yamaguchi, N., Existence of global strong solution to the micropolar fluid system in a bounded domain. Math. Meth. Appl. Sci., 2005, 28:1507-1526. MR2158216 (2008f:76055)

31. Yuan, B. Q., Regularity of weak solutions to magneto-micropolar fluid equations. Acta Math. Sci. Ser. B Engl. Ed., 2010, 30B.

32. Yuan, B. Q., Zhang, B., Blow-up criterion of strong solutions to the Navier-Stokes equations in Besov spaces with negative indices. J. Differential Equations, 2007, 242:1-10. MR2361099 (2009a:35189)

33. Zhou, Y., Regularity criteria in terms of pressure for the 3-D Navier-Stokes equations in a generic domain. Math. Ann., 2004, 328:173-192. MR2030374 (2004j:35229)

34. Zhou, Y., On regularity criteria in terms of pressure for the Navier-Stokes equations in $\mathbb{R}^{3}$. Proc. Amer. Math. Soc., 2006, 134:149-156. MR2170554 (2006i:35288)

35. Zhou, Y., On a regularity criterion in terms of the gradient of pressure for the Navier-Stokes equations in $\mathbb{R}^{N}$. Z. Angew. Math. Phys., 2006, 57:384-392. MR.2228171 (2007g:35179)

36. Zhou, Y., Remarks on regularities for the 3D MHD equations. Discrete Contin. Dyn. Syst., 2005, 12:881-886. MR2128731 (2005k:35344)

School of Mathematics and Information Science, Henan Polytechnic University, Henan 454000, People's Republic of China

E-mail address: bqyuan@hpu.edu.cn 